\title{
Regulation of research through research governance: within and beyond NSW Health
}

\author{
Geoffrey S. Bloom ${ }^{\mathrm{A}, \mathrm{C}}$ and Deborah Frew ${ }^{\mathrm{B}}$ \\ ${ }^{\mathrm{A}}$ Geoffrey Bloom \& Associates, Sydney \\ ${ }^{B}$ Policy Impact Program, Sax Institute, Sydney \\ ${ }^{\mathrm{C}}$ Corresponding author.Email: gbloom@gbloom.com.au
}

\begin{abstract}
Research governance takes a broad approach to the regulation of human research encompassing: (a) frameworks and systems over ad hoc policy making; (b) quality standards as well as regulatory requirements; and (c) definition of roles and responsibilities of all parties involved in research. The effective and consistent implementation of research governance plays a role in the promotion of quality research. NSW Health has recently issued several policies and procedures in relation to research governance. However, for regimes of research governance to achieve optimal effectiveness, they must be consistent with each other in both the public and private sectors and across Australian jurisdictions.
\end{abstract}

Research involving human participants is regulated in many ways and at many levels. Regulatory requirements apply through Commonwealth and state legislation, and deal with a range of matters such as the rules governing the conduct of clinical trials for therapeutic goods, radiation safety, use of human tissue samples and privacy of personal health information. ${ }^{1-7}$ However, in addition to this legislative regulation of research, a relatively new concept of research governance has been developed. Research governance is a wider concept than regulation as it is directed to organisational frameworks and quality standards rather than mere mandates and prohibitions. In this article, we aim to:

- explain the meaning of research governance and its importance

- describe recent developments in research governance within NSW Health

- present arguments as to why consistent research governance frameworks across Australia are desirable for the promotion of research.

\section{Research governance and its importance}

Research governance has been described as an organisational framework through which all research meets appropriate standards of quality, safety, privacy, risk management, financial management and ethical acceptability and where the roles, responsibilities and accountabilities of all those who play a part in research are specified. ${ }^{8}$ This definition is similar to those used elsewhere in Australian and United Kingdom (UK) guidelines and in the literature. ${ }^{9-11}$ This definition highlights three important concepts in research governance:

- frameworks and systems over ad hoc policy making

- quality standards and practices as well as regulatory requirements

- the definition of roles and responsibilities for all parties involved in research.

Implicit in these three points is the concept of consistency as an important element to be considered in the implementation of research governance frameworks in Australia.

\section{Frameworks and systems}

The concept of frameworks and systems is core to instigating a transparent and accountable approach to research governance. In the past, many research institutions dealt with the governance of research in an ad hoc manner. This is unsurprising, given that there were few high-level policies that either delineated appropriate research governance standards or required them to be imbedded in the practice of institutions or health systems. Although there are existing effective instruments in relation to certain aspects of research governance (e.g. the National Statement on Ethical Conduct in Human Research), there has been a lack of policy, or at least of transparency, in relation to other aspects of governance. ${ }^{9}$

\section{Standards and practices}

The concept of standards and practices is important, because it underpins the quality of research that is produced. Meeting regulatory requirements is clearly a necessary part of good research but, in itself, is not sufficient. Regulations can mandate or prohibit the undertaking of certain actions or require the establishment of certain review mechanisms. However, the achievement of high standards of quality and safety requires flexible instruments against which performance can be assessed and improved. It requires the application of expert opinion, judgement and peer assessment, rather than just a measurement of compliance or non-compliance. 


\section{Roles and responsibilities}

The concept of defining the roles and responsibilities of all parties is of crucial importance, as it recognises that all participants - including governments, health systems, institutions, various review bodies and researchers - have a role to play in ensuring high quality, safe and ethical research. In the past, there has been a tendency to conflate research governance and ethical review of research, so that some Human Research Ethics Committees (HRECs) have been required to play the role of both ethical reviewer and institutional gatekeeper. The proper implementation of research governance recognises that health systems, institutions and researchers all have roles in relation to the proper conduct of research and that it improves the ability of each player to fulfil their responsibilities efficiently and effectively if their roles are clearly defined.

\section{Consistency}

The question of what is meant by consistency in research governance is one that bears examination. There are many different types of research, governed by various legislation, guidelines, codes of practice and accepted standards. The requirements in relation to governance of clinical trials, for example, will be different to those for population health research. We do not suggest that these different types of research in different settings should be governed in exactly the same manner. What we do advocate is a basic set of underlying requirements that are consistent across institutions and jurisdictions in order that the following may occur:

1. there is a level of public accountability in ensuring that all factors relevant to the governance of a research project have been examined by an appropriate authority, regardless of where the research takes place

2. researchers are able to approach different institutions for approval of their research with the same, or a similar set of, information regarding their project, as opposed to producing a new set of information for each institution

3. the various stakeholders in the research endeavourincluding institutions, researchers and HRECs - are clear as to the role they play in relation to the governance of research, and that these roles are defined at a system level, not at the individual committee or institutional level.

\section{Research governance developments within NSW Health}

Most human research undertaken in Australia is conducted in hospitals, their associated universities and research institutes, many of which are public institutions. As New South Wales (NSW) is Australia's most populous state with the largest public hospital system, NSW Health policies are likely to apply to a significant proportion of all human research in Australia. ${ }^{11}$ In the past five years or more, NSW Health and other state government authorities have developed policies that have given organisational shape and substance to the concept of research governance. It is important for those involved in research within NSW Health to understand the policies that comprise the new research governance framework in this state.

\section{Frameworks and systems}

NSW Health has developed standard operating procedures for its HRECs. ${ }^{12}$ The standard operating procedures cover: objectives; functions; scope of responsibility; status of the HREC within the area health service; accountability of the HREC; membership; conduct; post-approval responsibilities and activities; and complaints and review. The aim of these policies is to save individual HRECs from establishing their own operational parameters, thereby allowing them to spend more time and resources on their core business of ethical review. These standard operating procedures also aim to enhance consistency of HREC operations across NSW Health.

NSW Health's recent policy and procedures to provide for single ethical and scientific review of multi-centre research aim to reduce duplication of HREC effort and make more efficient use of scientific and ethical expertise, and also to save time for proponents of research. ${ }^{13,14}$ The ethical review of research will be conducted by an accredited lead HREC and this single approval will serve as a sufficient ethical approval for the proposed research at any site within NSW Health.

The line between quality improvement and research can sometimes be difficult to draw. In either case, the activity may raise ethical issues beyond those occasioned by direct patient care. The relevant NSW Health guideline, which elaborates upon the national standard, aims to assist in determining whether a quality improvement exercise raises ethical risks to participants requiring review by an HREC. ${ }^{15,16}$

\section{Standards and practices}

The quality of the operations of NSW Health lead HRECs is now determined by accreditation standards. ${ }^{13,14}$ Each lead HREC is accredited in a specific type of research; for example, clinical trials or epidemiological research. Among other things, the accreditation standards provide for lead HRECs to be registered with the National Health and Medical Research Council (NHMRC), to meet NHMRC minimum membership requirements in terms of number of members and requisite expertise, to have sufficient resources and executive support, and to meet a maximum 60 day turnaround for research applications.

For research to be ethical it must be of sufficient scientific quality. NSW Health policy seeks to support standards of scientific review of clinical drug trials, including providing 
for review by a body other than the reviewing HREC, where scientific issues are beyond that HREC's expertise. ${ }^{17}$ The aim of this policy is to assist HRECs to comply with legal and administrative requirements under the Clinical Trial Exemption (CTX) and the Clinical Trial Notification (CTN) Schemes of the Therapeutic Goods Administration.

Clinical trial agreements are now generally standardised, with all commercially sponsored research conducted within NSW Health being considered in accordance with the Medicines Australia standard form clinical trial agreement, developed jointly by Medicines Australia, the Victorian Managed Insurance Authority and NSW Health, and in consultation with other Australian health departments. ${ }^{18}$ A similar agreement will be released to govern trials sponsored by contract research organisations and collaborative trial groups.

\section{Roles and responsibilities}

A major change aimed at shifting the governance burden associated with research away from HRECs is the advent of a policy requiring each research site or institution to consider and approve the operational dimensions of having research conducted within its facilities. ${ }^{19}$ A research governance officer, reporting to the institution rather than the HREC, is responsible for considering whether the use of the institution's resources (such as facilities, staff and equipment) is appropriate, whether the project adheres to the institution's site-specific policies (such as sign-offs from appropriate heads of department) and whether the institutionally based researchers involved in the project have the relevant training, expertise and experience. Research within an area health service may only begin once it has scientific and ethical approval by a duly constituted HREC and operational approval for each specific site within the area health service and the Chief Executive of the area health service, or delegate, has agreed to the commencement of the research.

Given that entities other than NSW Health are responsible for initiating and sometimes conducting research within NSW Health, it is necessary to define the roles and responsibilities applicable to insurance and indemnity for research. At the time of writing, NSW Health has released a draft policy for area health services to assist them with this crucial aspect of risk management and to standardise requirements throughout NSW Health. ${ }^{20}$

\section{Expanded horizons for research governance}

We have argued that research governance plays a role in promoting higher quality human research; has the potential to streamline the research approval process; and ensures that the main stakeholders in the research endeavour are vested with appropriate responsibilities. The NSW Health framework described above is but one example of a research governance system that could apply within
Australia. At present, it relates only to the NSW public health system and not to the private sector, the university sector or other Australian jurisdictions. Other Australian states and institutions have also implemented governance frameworks for human research. As yet, it is not possible to tell which of the (aspects of) various frameworks will prove superior. All other things being equal, it is likely that consistency in research governance across research settings will improve the effectiveness of research governance in any one setting. We advocate that this level of consistency should be national, for the following reason.

A great deal of research in Australia is undertaken on a national, multicentre basis. ${ }^{21,22}$ That is, it is conducted in multiple sites within different jurisdictions and across the public and private sectors. We have argued that consistency (as we have defined it) is important to achieving the aims of research governance. In the context of multicentre research, it is reasonable to expect that this consistency is best found at a national level. By extension, and given that much research in Australia is part of larger research projects spanning several countries, it is also desirable to seek to achieve consistency with the larger centres of research beyond Australia.

Australia has often been described as a good place to do research. ${ }^{21,23,24}$ Although this is undoubtedly the case, there are several factors that mitigate against Australia's ability to compete with other countries in maintaining and further developing a strong, vibrant and innovative research culture. Many of these factors are out of our control, such as geographic isolation, shortage of human research subjects and growth in study costs in comparison to other Asia-Pacific and emerging European nations. ${ }^{22}$ However, the regulatory and governance environment is amenable to control and can play a significant role in Australian and international decisions as to where to conduct research, particularly in relation to clinical trials. ${ }^{21,22}$

The NHMRC is currently engaged in a project to streamline ethical review of multicentre research nationally. ${ }^{25}$ This project is an example of the approach we advocate: ethical review of multicentre research is one part of research governance and the multicentre nature of this project implies the desirability of consistency in research governance across centres. It remains to be seen which (parts) of the developing regimes of research governance in Australia will prove most effective, including the regime within NSW Health. In our view, the degree to which one regime is consistent with others is, in itself, a significant element is its effectiveness.

\section{Conclusion}

The implementation of research governance frameworks is a valuable development in promoting high quality, safe and ethical research. Such a research governance framework 
is being developed within NSW Health. However, a national approach to research governance is desirable, and its value is likely to be enhanced if the approach is as consistent as possible across jurisdictions, institutions and public and private sectors.

Disclosure: Deborah Frew was Director, Health Research and Ethics Branch, NSW Department of Health at the time the policies discussed in this article were developed and played a substantial role in their content.

Geoffrey Bloom has provided legal and policy advice to the Health Research and Ethics Branch, on issues of research governance.

\section{References}

1. Therapeutic Goods Act 1989 (Cth).

2. Therapeutic Goods Regulation 1990 (Cth).

3. Radiation Control Act 1990 (NSW).

4. Radiation Control Regulation 2003 (NSW).

5. Human Tissue Act 1983 (NSW).

6. Health Records and Information Privacy Act 2002 (NSW).

7. Privacy Act 1988 (Cth).

8. National Health and Medical Research Council, Australian Research Council, Universities Australia. Australian code for the responsible conduct of research. Revision of the joint NHMRC/AVCC statement and guidelines on research practice. Canberra: Australian Government; 2007. Available from: http://www.nhmrc.gov.au/publications/synopses/r39syn.htm (Cited 17 July 2008.)

9. Australian Government (NHMRC, ARC), Australian Vice-Chancellors' Committee. National statement on ethical conduct in human research. Canberra: Australian Government; 2007. Available from: http://www.nhmrc.gov.au/publications/ synopses/e72syn.htm (Cited 17 July 2008.)

10. UK Department of Health, Social Services and Public Safety. Research governance framework for health and social care, 3rd edn. Belfast: Department of Health, Social Services \& Public Safety; 2005. Available from: http://www.centralservices agency.com/files/rdo_whats_new/file/RGF_061106.pdf (Cited 17 October 2008.)

11. Walsh MK, McNeil JJ, Breen KJ. Improving the governance of health research. Med J Aust 2005; 182(9): 468-71.

12. NSW Health. Human research ethics committees: operations manual for NSW Health HRECs, GL2005_059. North Sydney: NSW Health; 2005. Available from: http://www.health. nsw.gov.au/policies/gl/2005/GL2005_059.html (Cited 17 July 2008.)

13. NSW Health. Model for single ethical \& scientific review of multi-centre research, PD 2007_072. North Sydney: NSW Health; 2007. Available from: http://www.health.nsw.gov.au/ policies/pd/2007/PD2007_072.html (Cited 17 July 2008.)
14. NSW Health. Human research ethics committees: national ethics application form - application within NSW Health, PD2007_026. North Sydney: NSW Health; 2007. Available from: http://www.health.nsw.gov.au/policies/pd/2007/ PD2007_026.html (Cited 17 July 2008.)

15. NSW Health. Human research ethics committees - quality improvement \& ethical review: a practice guide for NSW, GL2007_020. North Sydney: NSW Health; 2007. Available from: http://www.health.nsw.gov.au/policies/g1/2007/ GL2007_020.html (Cited 17 July 2008.)

16. NHMRC. When does quality assurance in health care require independent ethical review - Advice to Institutions, Human Research Ethics Committees and Health Professionals. North Canberra: Commonwealth of Australia; 2003. Available from: http://www.nhmrc.gov.au/health_ethics/human/conduct/ guidelines/_files/e46.pdf (Cited 17 July 2008.)

17. NSW Health. Human research ethics committees: standards for scientific review of clinical trials, PD2007_035. North Sydney: NSW Health; 2007. Available from: http://www.health.nsw. gov.au/policies/pd/2007/PD2007_035.html (Cited 17 July 2008.)

18. NSW Health. Clinical research: standard clinical trial research agreement for NSW public health organisations - NSW Department of Health, PD2007_038. North Sydney: NSW Health; 2008. Available from: http:/www.health.nsw.gov.au/ policies/pd/2008/PD2008_039.html (Cited 17 July 2008.)

19. NSW Health. Research - Authorisation of proposals to conduct research on humans [Includes the Site Specific Assessment (SSA) Form \& Guidance document for completing the SSA Form], PD2007_043. North Sydney: NSW Health; 2007. Available from: http://www.health.nsw.gov.au/ policies/pd/2007/PD2007_043.html (Cited 17 July 2008.)

20. NSW Health. Clinical trials: risk management, insurance and indemnity, draft policy directive (for consultation). North Sydney: NSW Health; 2005.

21. Woolley K, Woolley M. Clinical trials in Australia-the FACTS. Good Clin Prac J 2003; 10(5): 13-6.

22. The Allen Consulting Group. Drivers of pharmaceutical industry investment; understanding Australia's competitive position. Available from: http:/www.medicinesaustralia.com.au/ pages/images/Drivers\%20of\%20Pharmaeceutical\%20Industry \%20Investment\%20FINAL20060915.pdf (Cited 17 July 2008.)

23. Mudge SJ. Australia: The number one place for clinical trials? App Clin Trials 2006; 15(9): 44-51.

24. The Economist Intelligence Unit. Benchmarking Study of the Australian and International Pharmaecuticals Industries. Canberra: Australian Government Department of Industry, Tourism and Resources; 2005. Available from: http://www.innovation.gov.au/General/Innov-PS/Documents/ Pharma_Benchmarking_Final_Sept20051007095438.pdf (Cited 17 July 2008.)

25. Australian Government, National Health and Medical Research Council. Harmonisation of Multi-centre Ethical Review (HoMER). Canberra: Australian Government; 2008. Available from: http://www.nhmrc.gov.au/health_ethics/ hrecs/homer.htm (Cited 22 October 2008.) 